\title{
A Combined Therapy Protocol to Improve Lower Extremity Muscle- Pumping Function and Lymphatic Drainage for Venous Ulcer Healing
}

Eduardo Simões Da Matta1*, Rodrigo Kikuchi², Clodoaldo Antônio De Sá ${ }^{3}$, Sinval Adalberto Rodrigues-Junior ${ }^{3}$ and Amanda Naiara Gustman ${ }^{1}$ ${ }^{1}$ Pró Circulação Clinic of Angiology and Vascular Surgery, Rua Coronel Passos Maia, 360, Ed. Policenter, Sala 101, zip code 89.820-000, Xanxerê, SC, Brazil ${ }^{2}$ Instituto de Excelência Vascular, Rua Itapeva, 202, $12^{\circ}$ andar, Zip code 01.332-000, São Paulo, SP, Brazil

${ }^{3}$ Health Sciences Postgraduate Program, Community University of the Region of Chapecó, Unochapecó, PO box 1141, Zip code 89809-000, Chapecó, SC, Brazil

\begin{abstract}
Objectives: To conduct a registry-based evaluation of a therapy protocol for chronic venous ulcers involving the improvement of lower extremity muscle-function along with lymphatic drainage and inelastic compression.

Method: We observed sixty-five patients with lower extremity venous ulcers secondary to chronic venous insufficiency until the end of treatment or the last available follow-up. The treatment protocol involved a series of sequential exercises with short-stretch bands performed thrice weekly: An hour of lymphatic drainage preceded each exercise session, associated with lymphomyokinetic passive activities using the RB3030 device. All patients underwent inelastic compression either with an Unna boot or with short-stretch bands. Perimetry was conducted at two different levels of the foot, and at five distinct levels of the leg, using a regular commercial tape. The ulcer wound was photographed along with an object with a pre-established, standard dimension. The area of each wound was then estimated through the Imagej software (National Institutes of Health) using a freehand drawing surrounding its border. Generalized linear models measured the association between therapy and outcomes, including the change in ulcer area, time-until-healing, and foot and ankle perimetry.

Results: Most of the patients were female, with $20 \%$ presenting a diagnosis of diabetes mellitus, $66.2 \%$ of hypertension, and $3.1 \%$ presenting signs of arterial insufficiency. Ulcer location was similarly distributed across sides $(23.1 \%$ vs. 21.5$)$, demonstrating similar percentages of previous deep vein thrombosis $(9.2 \%$ on the right and $6.2 \%$ on the left side). Average healing time was 37.24 days. Most ulcers (95.7\%) healed within the study period. Compared to their counterparts, patients with greater than 22 degrees of ankle range of motion presented an improved reduction in ulcer area size: $1.78(1.56,2.03)$ versus $2.85(2.23,3.64)$, respectively.
\end{abstract}

Conclusions: Therapies involving combined muscle strengthening, lymphatic drainage and compressive inelastic therapy should be part of chronic venous ulcer treatments.

Keywords: Venous stasis; Lymphatic system; Lymphatic medicine; Leg ulcers; Compression; Lymphatic drainage; Muscle pump function

\section{Introduction}

Venous ulcers are the most common leg ulcers, with a high morbidity and extended length of care, frequently leading to increased healthcare expenditures and a reduced quality of life. Venous ulcers result from chronic venous hypertension which in turn results from primary or secondary venous disease (post-thrombotic, post-surgical or after injury), followed by an inflammatory reaction leading to hypoxia and cellular death [1]. Dysfunction of the calf muscle pump has demonstrated to be a risk factor for venous ulcers [2]. However, current guidelines for the management of venous ulcers do not regularly include measures to improve lower extremity muscle pump function focused on all segments including foot, calf, and thigh.

Evidence-based standard treatment options for venous ulcers include conservative management (compression therapy, leg elevation, and dressings), mechanical treatment (vacuum-assisted closure), medications, as well as surgical treatment. Compression therapy is considered to be the gold-standard treatment for venous ulcers and chronic venous insufficiency. Surgical interventions are reserved for recalcitrant ulcers [3-6]. The prolonged use of inelastic compression, however, leads to a decrease in the extremity diameter as a result of a reduction in edema as well as the loss of muscle mass [7]. Also, compression therapy leads to slower healing as well as high recurrence rates, especially when compression is not maintained after healing $[8,9]$. Long-term treatment with compressive therapy is not always feasible as a function of comorbidities including pain, arthritis, wound drainage, obesity, contact dermatitis, as well as illnesses such as arterial disease and decompensated heart failure [8]. Given these limitations, exploring other treatment options is important.

Although not extensively studied, venous ulcer care involving the improvement of calf muscle pump function could be beneficial given the underlying pathophysiology of this condition. For example, muscle wasting and decreased ankle range of motion have been implicated in the pathogenesis of venous ulceration [10], calf muscle pump failure being a common finding in patients with venous ulcers [11]. This failure can, however, be prevented by an improvement in calf muscle pump function achieved through physical exercise [12]. Furthermore, ankle range of motion is an important component in the activation of

*Corresponding author: Eduardo Simões da Matta, Medical Director - Pró Circulação Clinic of Angiology and Vascular Surgery, Rua Coronel Passos Maia 360, Ed. Policenter, Sala 101, Centro, CEP 89820-000, Xanxerê, Santa Catarina Brazil, Tel: +55-49-34339050; E-mail: procirculacao@gmail.com

Received October 27, 2017; Accepted November 16, 2017; Published November 23, 2017

Citation: Matta ESD, Kikuchi R, De Sá CA, Rodrigues-Junior SA, Gustman AN (2017) A Combined Therapy Protocol to Improve Lower Extremity Muscle-Pumping Function and Lymphatic Drainage for Venous Ulcer Healing. J Vasc Med Surg 5 347. doi: 10.4172/2329-6925.1000347

Copyright: ( 2017 Matta ESD, et al. This is an open-access article distributed under the terms of the Creative Commons Attribution License, which permits unrestricted use, distribution, and reproduction in any medium, provided the original author and source are credited. 
the calf muscle pump [13], based on a previously reported relationship between venous ulcer healing and the improvement of ankle range of motion for plantar flexion and inversion [14]. Also, we observed that ankle joint range of the movement might also be affected by lymphatic drainage of ulcerated limbs, although this can be reversed through lymphatic drainage [15]. Therefore, we could associate the management of chronic venous ulcers with a focus on combined improvement of muscle function and lymphatic drainage with better outcomes. To our knowledge, however, no previous study has assessed the impact of improved muscle function and ankle range of motion on venous ulcer healing.

In an attempt to address this gap in the literature, this study aims to assess the effects of improved lower extremity muscle function combined with lymphatic drainage and inelastic compression on venous ulcer healing. Of importance, our protocol focuses on all lower extremity segments including the foot, calf, and thigh.

\section{Methods}

\section{Study design}

This study is an observational, registry-based study to evaluate the association between a treatment to enhance lower extremity-pumping function combined with lymphatic drainage and inelastic compression, and its effect on venous ulcer healing. The Reporting of Observational Studies in Epidemiology (STROBE) statement was used to describe this study [16].

\section{Setting}

We collected data from patients who were clinically diagnosed with venous ulcers at the Pro Circulation Clinic of Angiology and Vascular Surgery, Xanxerê/SC, Brazil. Patient follow-up commenced on the initial consultation date to the time of ulcer healing, or the last available follow-up. Participant accrual occurred between January of 2012 and March of 2016.

\section{Ethics}

The Institutional Review Board of the Community University of the Chapeco Region, Brazil approved our study (protocol number 60269516.7.0000.0116).

\section{Participants}

We included 65 patients, with 92 lower limb venous ulcers secondary to chronic venous insufficiency, and excluded patients who did not complete treatment as well as those with a diagnosis of arterial insufficiency defined as an Ankle Brachial Index $<0.8$.

\section{Interventions}

Our treatment protocol involved a series of sequential exercises performed three times a week: A one-hour lymphatic drainage preceded each exercise session, associated with myolymphokinetic activities including day-to-day activities or exercises resulting in a volumetric reduction of the limb using the RB3030 passive mechanical device (RB Equipamentos Médicos LTDA.-ME/São José do Rio Preto - SP). This device is a lymphatic-drainage apparatus with a low-rotation engine which performs passive flexion and stretching exercise sessions of the lower extremities for one hour daily [17]. All patients also underwent inelastic compression either with an Unna boot or with short-stretch bands. Our exercise protocol involved a warm-up session using an ergometric bicycle for five minutes at a self-selected speed, followed by strengthening exercises with maximum load, developed in three series of 15 repetitions each, with a one-minute interval between series. We progressively adjusted the number of sets and weights during the first four to six exercise sessions. Training modalities included:

- Flexion and extension of the ankle joint performed in standing position, with the distal third of the foot lying on a 15-centimeter high platform, with an optional weight increase obtained through additional lead-belts.

- Hip adduction and abduction in lateral decubitus, with a possible increase in weight through ankle weights.

- Squatting against the wall using a heavy ball, with possible additional weight.

- Ankle dorsi and plantar flexion with weight transfer from the heel to the forefoot in a sitting position, and with weight on top of the thigh.

- Carrying an additional weight while going up and downstairs.

We conducted compressive therapy through short stretch bands, performed by a trained physical therapist three times per week. Some patients received an Unna boot for seven days, applied by a trained, certified nurse usually after the edema had decreased as a result of the protocol mentioned above. Ulcer care was provided to all patients once or twice a week by a certified nurse, following principles of maintaining a hydrated surface between the ulcer and the dressing, while also performing a debridement.

Aligned with the previous description, we made use of an ipsilateral range of motion as a proxy for the intervention and measured dorsiflexion and plantar flexion through goniometry. Specifically, passive ankle motion was performed [14], with the fixed arm of the goniometer located on the leg axis, aligning the movable arm with the foot axis. We performed all procedures in an office setting by trained physical therapists. A selected group of patients had the goniometry performed twice by two independent physical therapists to assess the inter-observer reliability of these metrics. Perimetry was conducted at two different levels of the foot, and at five distinct levels of the leg, using a regular commercial tape. No pressure was applied to these measurements to avoid affecting their precision. We conducted the first-foot measurement five centimeters proximal to the base of the first proximal phalanx of the second toe, and the second measurement five centimeters proximal to the first measure. The first leg measurement was performed five centimeters above the inferior border of the lateral malleolus, and then followed at every five centimeters to a total of six to eight measurements depending on the leg height.

Ulcer size was measured using a standard protocol: First, we photographed the wounds at a normal distance along with an object with a pre-established, standard dimension. The area of each wound was then estimated through the Imagej software (National Institutes of Health) using a freehand drawing surrounding its border, with software calibration based on the pre-established dimensions of the background image. We assessed a subset of 20 images by two independent observers to estimate inter-observer reliability.

\section{Potential confounding variables}

Using clinical judgment combined with previous evidence from the literature, we selected age and diabetes as potential confounders [18].

\section{Statistical methods}

We evaluated variables through exploratory analysis, assessing the 
frequency, percentage and near-zero variance for categorical variables, distribution for numeric variables, and missing values and patterns of all variables [19]. Also, to guide bivariate plot inspection, we ran a MINE algorithm [20]. Generalized linear models were used to measure the association between range-of-motion and outcome variables. Since the ulcer area was skewed to the right, models were performed using log-transformed variables. Time-until-ulcer healing was evaluated using a combination of Kaplan-Meier plots, log-rank tests, and Cox Proportional Hazard models. We assessed inter-observer reliability for the ulcer measurement through intra-class correlation coefficients with $95 \%$ confidence intervals and performed all analyses with the $\mathrm{R}$ statistical language [21].

\section{Results}

\section{Sample characteristics}

Most patients were female, with $20 \%$ having a diagnosis of diabetes mellitus, $66.2 \%$ of hypertension, and only $3.1 \%$ having signs of arterial insufficiency. Ulcer location was similarly distributed across sides ( $23.1 \%$ on the right and 21.5 on the left side), also demonstrating similar percentages of previous deep vein thrombosis (DVT) $(9.2 \%$ on the right and $6.2 \%$ on the left side). No statistically significant differences were found between those below and above mean age (Table 1). The comparison of ulcer area measures between two observers reached an intra-class correlation coefficient of 0.82 and therefore considered reliable.

Since a patient could have more than one ulcer, Table 2 describes the study sample at the ulcer level, stratified by median age ( $\mathrm{n}=71$ years). Most ulcers were followed-up for 73.5 days, which also represents their average healing time. A total of $95.7 \%$ ulcers healed during the study period, with ulcers equally distributed about the site $(52.2 \%$ on the left and $47.8 \%$ on the left). Most ulcers presenting satellite sites presented a total of two isolated ulcers (17.4\%). The average initial surface area was 22.8 square centimeters, reduced to a final average ulcer area of 0.22 square centimeters since most ulcers healed during the study period. Ipsilateral change in range-of-motion after therapy had a mean of 15.7 degrees, with overall lower extremity perimetry (the sum of all foot and leg perimetry measures) reducing from 60.4 to 56.2 centimeters with treatment. We found no statistically significant changes between those below and above the median age.

All but four ulcers healed within the study period, the overall survival thus demonstrated under Figure 1. When evaluating timeuntil-ulcer healing adjusted for comorbidities including diabetes and hypertension, we found no statistically significant differences between groups (Figures 2 and 3 ).

To evaluate the association between ankle range of motion and reduction in ulcer area, we used the 80th percentile of the range of motion ( $n=22$ degrees). Since four patients presented ulcer areas of over 100 square centimeters, the distribution was not normal, and therefore these patients were excluded from this analysis. We associated patients with a range of motion above this level with a significantly greater ulcer area reduction than those with a range of the movement lower than 22 degrees: 2.85 square centimeters (2.23-3.64) versus 1.78

\begin{tabular}{|c|c|c|c|c|}
\hline Variable [Missing] & Total (65) & Age $\leq 72(33)$ & Age $>72(32)$ & $\mathbf{p}$ \\
\hline Female [0] & $51(78.5 \%)$ & $24(36.9 \%)$ & $27(41.5 \%)$ & 0.401 \\
\hline Diabetes [0] & $13(20 \%)$ & $5(7.69 \%)$ & $8(12.3 \%)$ & 0.495 \\
\hline Hypertension [0] & $43(66.2 \%)$ & $23(35.4 \%)$ & $20(30.8 \%)$ & 0.726 \\
\hline Arterial Insufficiency [0] & $2(3.08 \%)$ & $1(1.54 \%)$ & $1(1.54 \%)$ & 1 \\
\hline Right Varicose Surgery [0] & $15(23.1 \%)$ & $6(9.23 \%)$ & $9(13.8 \%)$ & 0.511 \\
\hline Left Varicose Surgery [0] & $14(21.5 \%)$ & $9(13.8 \%)$ & $5(7.69 \%)$ & 0.401 \\
\hline Right DVT [0] & $6(9.23 \%)$ & $5(7.69 \%)$ & $1(1.54 \%)$ & 0.213 \\
\hline Left DVT [0] & $4(6.15 \%)$ & $1(1.54 \%)$ & $3(4.62 \%)$ & 0.584 \\
\hline
\end{tabular}

Table 1: Patient sample stratified by median age.

\begin{tabular}{|c|c|c|c|c|}
\hline Variable [Missing] & Total (92) & Age $\leq 71(48)$ & Age $>71(44)$ & p \\
\hline Follow-up days [0] & $73.5( \pm 79.9)$ & $69.2( \pm 80.2)$ & $78.3( \pm 80.3)$ & 0.589 \\
\hline Ulcer onset duration (months) [13] & $26( \pm 63.4)$ & $25.9( \pm 33.7)$ & $26( \pm 81.5)$ & 0.991 \\
\hline Healed ulcer [0] & $88(95.7 \%)$ & $47(51.1 \%)$ & $41(44.6 \%)$ & 0.548 \\
\hline Ulcer side [0] & & & & 0.149 \\
\hline Left & $48(52.2 \%)$ & $29(60.4 \%)$ & $19(43.2 \%)$ & \\
\hline Right & $44(47.8 \%)$ & $19(39.6 \%)$ & $25(56.8 \%)$ & \\
\hline Ulcer satellites [66] & & & & 0.166 \\
\hline 2 & $16(17.4 \%)$ & $8(57.1 \%)$ & $8(66.7 \%)$ & \\
\hline 3 & $5(5.43 \%)$ & $2(14.3 \%)$ & $3(25 \%)$ & \\
\hline 4 & $1(1.09 \%)$ & $0(0 \%)$ & $1(8.33 \%)$ & \\
\hline 5 & $4(4.35 \%)$ & $4(28.6 \%)$ & $0(0 \%)$ & \\
\hline Surface area at initial visit [0] & $22.8( \pm 94.4)$ & $35.1( \pm 129)$ & $9.27( \pm 20.2)$ & 0.176 \\
\hline Surface area at final visit [0] & $0.22( \pm 1.88)$ & $0.37( \pm 2.6)$ & $0.0417( \pm 0.17)$ & 0.38 \\
\hline Ipsilateral range motion change [4] & $15.7( \pm 9.64)$ & $16( \pm 10.2)$ & $15.2( \pm 9.07)$ & 0.69 \\
\hline Foot perimetry at initial visit [55] & $53.8( \pm 5.61)$ & $56( \pm 6.67)$ & $51.6( \pm 3.35)$ & 0.02 \\
\hline Foot perimetry at final visit [56] & $50.8( \pm 3.91)$ & $52.1( \pm 4.6)$ & $49.4( \pm 2.5)$ & 0.035 \\
\hline Leg perimetry at initial visit [1] & $60.4( \pm 19.2)$ & $68.3( \pm 23)$ & $51.6( \pm 6.9)$ & $<0.001$ \\
\hline Leg perimetry at final visit [2] & $56.2( \pm 16.5)$ & $63.2( \pm 19.4)$ & $48.2( \pm 6.13)$ & $<0.001$ \\
\hline Perimetry at initial visit [55] & $114( \pm 26.6)$ & $129( \pm 31.9)$ & $101( \pm 7.54)$ & 0.002 \\
\hline Perimetry at final visit [56] & $106( \pm 18.2)$ & $116( \pm 20)$ & $95.1( \pm 6.88)$ & $<0.001$ \\
\hline
\end{tabular}

Table 2: Sample description at the ulcer level. 


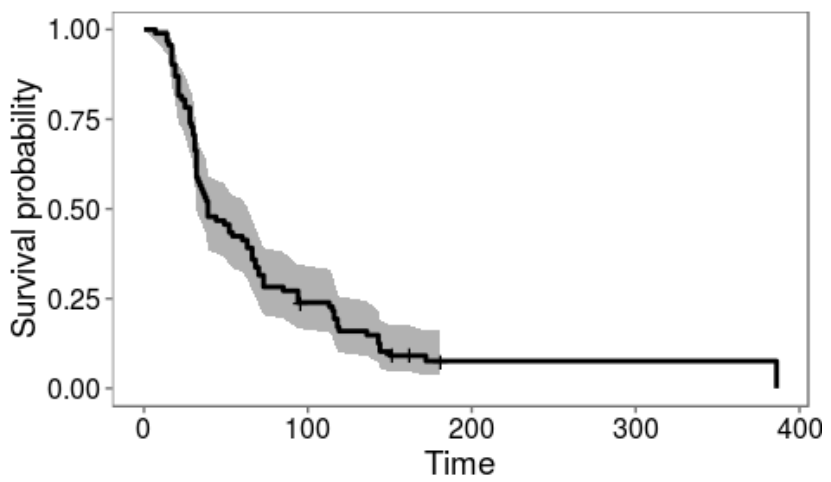

Figure 1: Kaplan-Meier curves with time to heal in all patients.

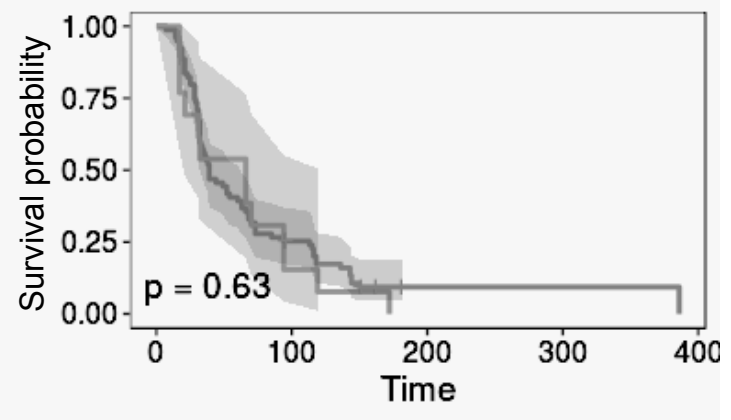

Figure 2: Kaplan-Meier curves with time to heal by diabetes.

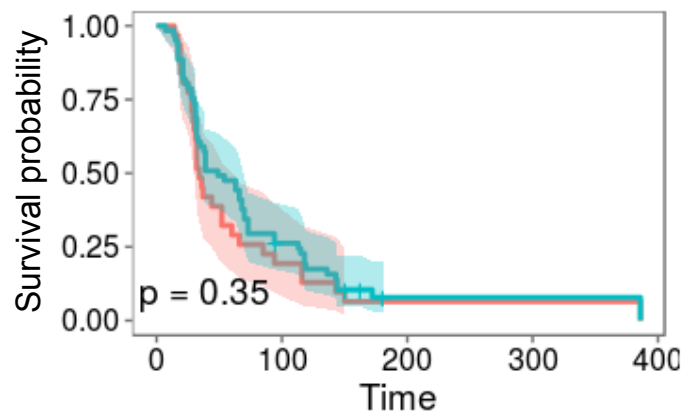

Figure 3: Kaplan Meier curves with time to heal by hypertension status.

square centimeters (1.56-2.03). However, patients with increased range of motion had no significant change in their final foot, leg or lower extremity perimetry between the initial and final visits. These results remained consistent when evaluating results adjusted for confounding factors. These results were also robust when evaluating not only the 80th percentile threshold, but the overall association between range of motion and reduction in ulcer area through a scatterplot $(\mathrm{p}<0.001)$ (Figure 4). Despite not having a statistically significant association, post-treatment perimetry measures were consistently lower than their initial counterparts (Figure 5).

In spite of its previously demonstrated association with reduction in ulcer area, there was no association between ankle range of motion and time until ulcer healing, for both the crude and the adjusted associations (Table 3).

\begin{tabular}{|c|c|}
\hline & Follow- up days \\
\hline Crude & $1.05(0.63,1.76)$ \\
\hline Adjusted & $1.06(0.63,1.79)$ \\
\hline
\end{tabular}

Table 3: Crude and adjusted survival analysis for patients with more or less than 22 degrees of motion.

\section{Discussion}

To the best of our knowledge, this is the first article describing an intense therapy protocol combining muscle strengthening for the entire lower extremity rather than just the calf, lymphatic drainage and inelastic compression in the treatment of chronic venous ulcers.

It was demonstrated that the average healing time across the entire sample was 37.24 days, with nearly all ulcers (95.7\%) in our series healing within the study period. Ankle range of motion at the 80th percentile (22 degrees) was statistically associated with ulcer size reduction, $1.78(1.56,2.03)$ for those with less than 22 degrees of motion, versus $2.85(2.23,3.64)$ for those with 22 degrees or greater, but not with a reduction in perimetry or time-until-ulcer healing.

Our results were promising when compared with those of compression therapy in the literature, the standard treatment for venous leg ulcers. Some studies evaluating compressive techniques reported greater healing times than those found in our sample or included subjects with smaller ulcer sizes. For instance, a trial comparing cohesive short stretch versus four-layer bandaging reported healing rates of $56 \%$ at 12 weeks in both groups [22]. Another study evaluating multilayer bandaging reported the median healing time in the treatment group at 133 days [23]. Finally, a study comparing a twocomponent Compression system with zinc paste bandages reported median healing time around 50 days for both techniques, but with a median ulcer surface area of 7.5 square centimeters, smaller than the 22.75 square centimeters found in our sample [24,25]. Regarding factors affecting time-until-healing, the complete healing of long-standing, large venous leg ulcers has been demonstrated to be independently associated with the ablation of incompetent superficial veins, as well as with the percentage of wound area reduction after the first four weeks of treatment [26]. Although our study sample does not present a control group, our relatively short healing time might relate to our protocol combining muscle strengthening and lymphatic drainage. Also, we described impaired lymphatic function as being associated with both venous leg ulcers and chronic venous hypertension, ultimately strengthening the likelihood that our results might be connected to our implemented therapeutic intervention [27]. While another critical factor affecting healing time is ulcer size, the current literature does not seem to agree on size thresholds. Ranging from observational studies that most closely mimic a real-world clinical practice but present issues with confounding, to randomized trials with more homogeneous patient samples that might not represent the actual daily practice encountered by most providers, this likely results from a significant heterogeneity in patient samples. Given these limitations, ulcer size associated with delayed healing has varied from over 10 to 20 square centimeters [28-32]. For studies using diameters as a metric, some have established the cut-point at 10 centimeters [33]. In contrast, small ulcers of 1 centimeter or less, were more likely to heal within a short period [34]. Moreover, several studies with relatively small samples or high-sample heterogeneity have failed to demonstrate an association between size and time to heal $[35,36]$. Finally, when evaluating risk factors related to the failure of compression therapy in treating venous leg ulcers, some studies demonstrated that factors such as high BMI $\left(>33 \mathrm{~kg} / \mathrm{m}^{2}\right)$, short walking distance during the day $(<200$ meters), a 


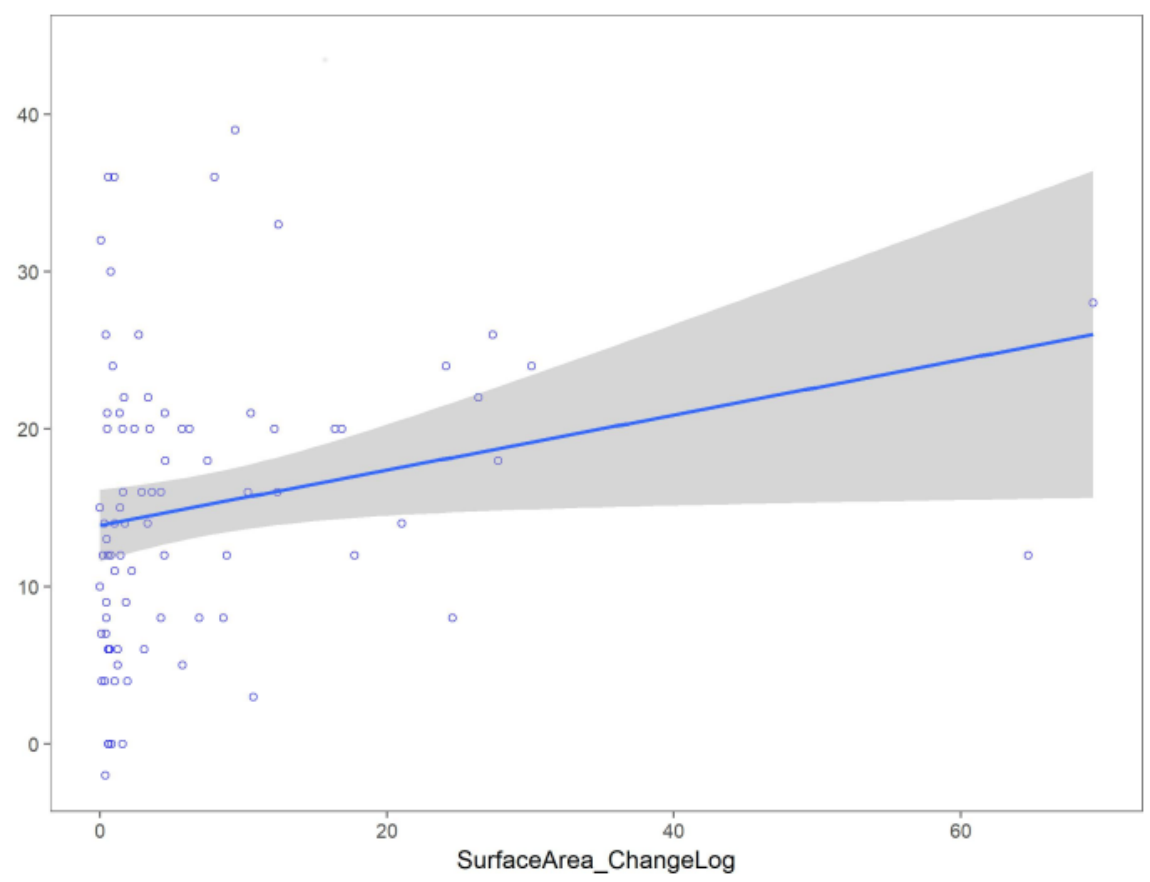

Figure 4: Scatterplot measuring association between ankle range of motion and reduction in ulcer area.

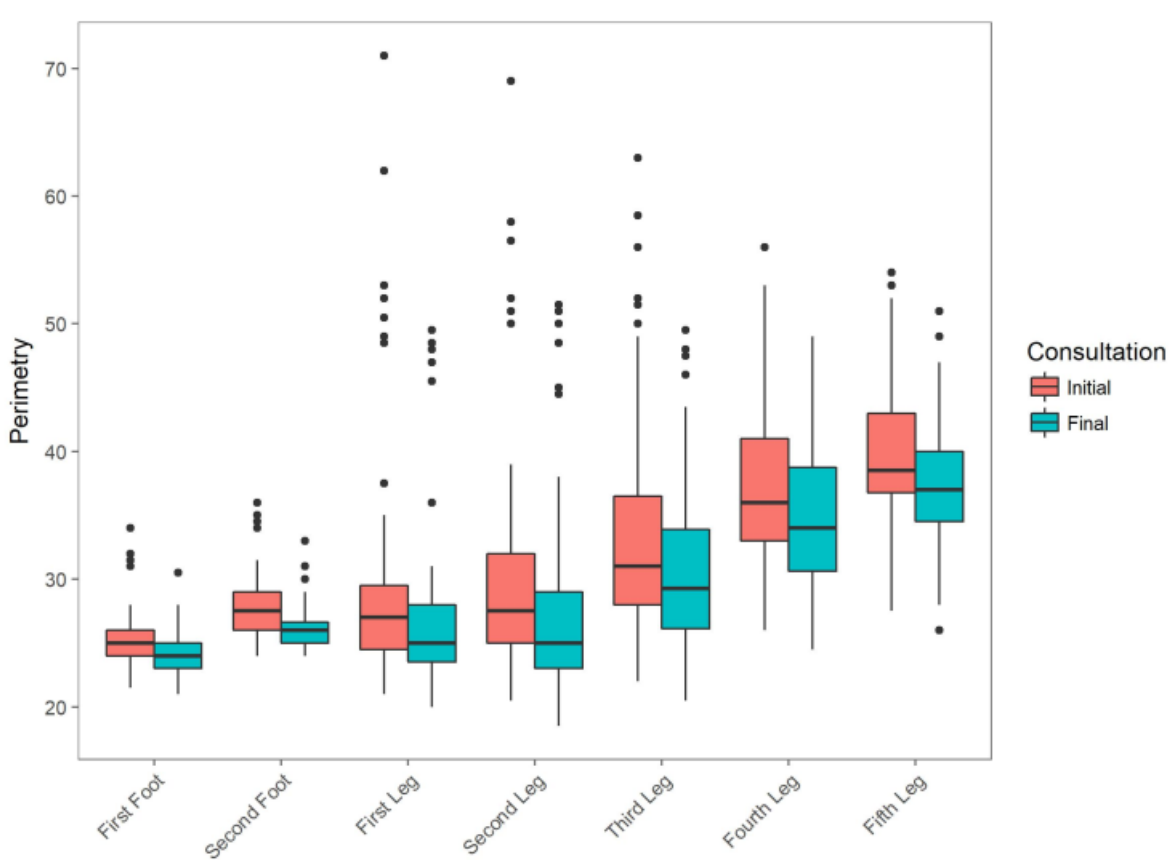

Figure 5: Comparison between perimetry values at the beginning and end of treatment.

history of wound debridement, and deep ulcers ( $>2$ centimeters) were indicators of slow healing [32].

Exercise has been demonstrated to increase calf muscle pump function significantly, notably the ejection fraction, resulting in a decrease in residual fraction through the rise in all endurance parameters [12]. During contraction, lower extremity muscles generate high pressure on the deep venous system, causing blood to return upwards. During muscle relaxation, the pressure falls, and the venous system is replenished, ultimately generating a mechanism that will continually drain the lower extremity. One-way valves contribute to this mechanism in that when properly functioning, they prevent venous reflux during both muscle contraction and relaxation phases. Although the calf pump is likely the most important, the thigh (quadriceps, sartorius and hamstring pumps), and foot pumps equally contribute to a well-functioning pumping system. Accordingly, gait efficiency, as well as a proper functioning of the venous drainage system, is dependent 
on the integrated functioning of all components. The foot pump, in particular, is not solely dependent on muscle function but can be active even with passive motion, as demonstrated among paralyzed limbs. It appears that the underlying mechanism relates to the stretching of the plantar veins during weight bearing, ultimately leading to the ejection of blood [37].

High rates of reflux and high residual volumes, independent of the site of reflux have characterized ulcerated limbs [38], thus establishing the connection between venous ulceration and isolated superficial venous system incompetence [38,39]. Given this underlying mechanism, muscle-strengthening programs including physical exercise have been described to improve calf muscle function in patients with chronic venous ulcers [12]. Specifically, isotonic muscle exercises are known to improve muscular endurance, efficacy, and power, ultimately leading to an overall improvement in the hemodynamic status of lower extremities with venous ulceration [40]. Venous insufficiency is also recognized as a necessary but insufficient condition to cause ulceration, and a deficiency of the calf muscle pump mechanism is significant in the ability to heal different venous ulceration conditions [2]. Finally, reduced ankle range of motion has been demonstrated to be a major factor in the development of chronic venous ulcer conditions and venous hypertension, not only leading to reduced ankle motion but also directly associated with the clinical severity of venous disease [41]. It would thus be natural to hypothesize that an increase in the range of motion could potentially contribute toward better outcomes in the treatment of chronic venous ulcers.

It is also worth mentioning that the interface pressure of a bandage system is dependent on the muscle forces generated inside the wrapped area [42]. Some authors reported that compression pressures of more than $50 \mathrm{mmHg}$ in orthostasis are necessary to achieve a significant reduction in venous hypertension among patients with chronic venous insufficiency, leading to effective ulcer healing. However, even if the system provides adequate pressure levels in supine position, these may not be sufficient for ulcer healing when in an upright position, depending on individual muscle strength levels [43]. This point is particularly important for the elderly since venous disease reaches its prevalence peak among those between 60 and 80 years of age [44]. This group of individuals is particularly predisposed to sarcopenia, especially when other risk factors for muscle mass loss are present, such as obesity, insulin resistance, and female gender. Improvements in the conditioning of the calf muscle system might thus be beneficial to the venous circulatory system, ultimately leading to an increase in the quality of life [45].

Abnormal lymphatic drainage has been reported to increase the severity of venous ulcer disease [46,47]. One study evaluated lymphatic drainage in a series of patients with unilateral leg ulcers, comparing it with the contralateral healthy leg and noting a decrease in lymphatic function in the leg with venous ulcers [48]. Another study constituting an essential component in the pathogenesis of this condition reported that chronic venous ulcers usually present a significant increase in edema focused on the papillary dermis, and further corroborated these results [49]. These findings have led some authors to conclude that impaired lymphatic drainage may be as important as venous pathology in chronic venous ulcers [27,48]. Accordingly, consensus guidelines have emphasized the importance of lymphedema investigation [50]. Nevertheless, when it comes to treatment, most guidelines will emphasize measures involving compressive stockings rather than the role of active and passive motion of the lower extremity. This is surprising, as active extremity movement has been demonstrated to contribute toward lymphatic drainage [51]
Despite filling an important gap in the literature, our study does have limitations usually associated with a case series design. First, we did not have a control group with patients undergoing different treatment options, limiting us to compare this intervention against a standard of care which frequently involves the treatment directed at local, rather than pump-related factors. Second, we did not have a measure of muscle strength. While foot and leg perimetry could have been considered an indirect measure of muscle strength, this metric is significantly confounded by the presence of edema. An ideal test would involve the use of magnetic resonance or other laboratory methods that can reliably assess muscle strength. These will be addressed in a future study involving multiple sites. Third, we did not include selfreported measures of quality of life or dysfunction which constitute an important metric, in that they take into account a linear patient perspective which is apparently missing when we use only providerdriven measures. The absence of these measures from our study primarily resulted from logistical limitations, since the inclusion of selfreported questionnaires would significantly increase the complexity of data collection for our registry. Fourth, given logistic limitations in our study, we performed lymphatic drainage but did not assess the presence of lymphedema through the usual pitting test, Stemmer sign, or imaging with lymphoscintigraphy. Lastly, we failed to identify the causes of the ulcer as resulting from superficial and deep venous insufficiency. Despite this limitation, compression therapy has been demonstrated to be the treatment of both conditions $[24,43,52]$.

In summary, our study points toward a potential role in the combined use of muscle strengthening, lymphatic drainage, inelastic compression of the lower extremity as a whole and local skin measures in the treatment of chronic venous ulcers. Future studies should conduct a comparison of each of these therapeutic modalities to evaluate the contribution of individual components toward ulcer healing.

\section{References}

1. Chatterjee SS (2012) Venous ulcers of the lower limb: Where do we stand? Indian J Plast Surg 45: 266-274.

2. Araki CT, Back TL, Padberg FT, Thompson PN, Jamil Z, et al. (1994) The significance of calf muscle pump function in venous ulceration. J Vasc Surg 20: $872-877$

3. Collins L, Seraj S (2010) Diagnosis and treatment of venous ulcers. Am Fam Physician 81: 989-996

4. O'Donnell TF, Passman MA, Marston WA, Ennis WJ, Dalsing M, et al. (2014) Management of venous leg ulcers: Clinical practice guidelines of the society for vascular surgery and the American venous forum. Journal of Vascular Surgery 60: 3-59.

5. Mosti G, De Maeseneer M, Cavezzi A, Parsi K, Morrison N, et al. (2015) Society for Vascular Surgery and American Venous Forum Guidelines on the management of venous leg ulcers: The point of view of the International Union of Phlebology. Int Angiol 34: 202-218.

6. Carvalho MR de (2015) Comparison of outcomes in patients with venous leg ulcers treated with compression therapy alone versus combination of surgery and compression therapy: A systematic review. J Wound Ostomy Continence Nurs 42: 42-46.

7. Flügge C, Hollmann W, Hettinger T, Rüter E (1971) Über den einfluß einer längeren benutzung von kompressionsstrümpfen auf die kraft der beinmuskulatur. Sportarzt u Sportmed 22: 287-290.

8. Raju S, Neglén $P$ (2009) Chronic Venous Insufficiency and Varicose Veins. N Engl J Med 360: 2319-2327.

9. Whiddon LL (2007) The treatment of venous ulcers of the lower extremities Proc (Bayl Univ Med Cent) 20: 363-366.

10. Davies JA, Bull RH, Farrelly IJ, Wakelin MJ (2007) A home-based exercise programme improves ankle range of motion in long-term venous ulcer patients. Phlebology 22: 86-89. 
Citation: Matta ESD, Kikuchi R, De Sá CA, Rodrigues-Junior SA, Gustman AN (2017) A Combined Therapy Protocol to Improve Lower Extremity Muscle-Pumping Function and Lymphatic Drainage for Venous Ulcer Healing. J Vasc Med Surg 5: 347. doi: 10.4172/2329-6925.1000347

11. Simka M (2004) Calf muscle pump dysfunction in the patients with severe chronic venous insufficiency. Phlebolymphology 47: 299-303.

12. Yang D, Vandongen YK, Stacey MC (1999) Effect of exercise on calf muscle pump function in patients with chronic venous disease. Br J Surg 86: 338-341.

13. O'Brien JA, Edwards HE, Finlayson KJ, Kerr GK (2012) Understanding the relationships between the calf muscle pump, ankle range of motion and healing for adults with venous leg ulcers: A review of the literature. Wound Practice \& Research 20: 80-85.

14. Yim E, Richmond NA, Baquerizo K, Van Driessche F, Slade HB, et al. (2014) The effect of ankle range of motion on venous ulcer healing rates. Wound Repair Regen 22: 492-496.

15. Pereira de Godoy JM, Braile DM, Fátima Guerreiro Godoy M de (2008) Lymph drainage in patients with joint immobility due to chronic ulcerated lesions. Phlebology 23: 32-34.

16. Elm E Von, Altman DG, Egger M, Pocock SJ, Gøtzsche PC, et al. (2007) The Strengthening the Reporting of Observational Studies in Epidemiology (STROBE) Statement: Guidelines for Reporting Observational Studies. Ann Intern Med 147: 573-577.

17. Godoy JMP de, Godoy M de FG de (2010) Godoy \& Godoy technique in the treatment of lymphedema for under-privileged populations. International Journal of Medical Sciences 7: 68-71.

18. Lee PH (2014) Should we adjust for a confounder if empirical and theoretical criteria yield contradictory results? A simulation study. Sci Rep 4: 6085.

19. Kuhn M, Johnson K (2013) Applied predictive modeling. Springer, ISBN-13.

20. Reshef DN, Reshef YA, Finucane HK, Grossman SR, McVean G, et al. (2011) Detecting novel associations in large data sets. Science 334: 1518-1524.

21. R Core Team (2015) R: A language and environment for statistical computing $R$ Foundation for Statistical Computing.

22. Franks PJ, Moody M, Moffatt CJ, Martin R, Blewett R, et al. (2004) Randomized trial of cohesive short-stretch versus four-layer bandaging in the management of venous ulceration. Wound Repair Regen 12: 157-162.

23. Milic DJ, Zivic SS, Bogdanovic DC, Perisic ZD, Milosevic ZD, et al. (2007) A randomized trial of the tubulcus multilayer bandaging system in the treatment of extensive venous ulcers. J Vasc Surg 46: 750-755.

24. Mosti G, Crespi A, Mattaliano V (2011) Comparison between a new, twocomponent compression system with zinc paste bandages for leg ulcer healing: A prospective, multicenter, randomized, controlled trial monitoring sub-bandage pressures. Wounds 23: 126-134.

25. Phillips TJ, Machado F, Trout R, Porter J, Olin J, et al. (2000) Prognostic indicators in venous ulcers. J Am Acad Dermatol 43: 627-630.

26. Chaby G, Senet P, Ganry O, Caudron A, Thuillier D, et al. (2013) Prognostic factors associated with healing of venous leg ulcers: A multicentre, prospective, cohort study. Br J Dermatol 169: 1106-1113.

27. Bull RH, Gane JN, Evans JE, Joseph AE, Mortimer PS (1993) Abnormal lymph drainage in patients with chronic venous leg ulcers. J Am Acad Dermatol 28: 585-590.

28. Franks PJ, Bosanquet N, Connolly M, Oldroyd MI, Moffatt CJ, et al. (1995) Venous ulcer healing: Effect of socioeconomic factors in London. J Epidemiol Community Health 49: 385-388.

29. Margolis DJ, Allen-Taylor L, Hoffstad O, Berlin JA (2004) The accuracy of venous leg ulcer prognostic models in a wound care system. Wound Repair Regen 12: 163-168.

30. Margolis DJ, Berlin JA, Strom BL (1999) Risk factors associated with the failure of a venous leg ulcer to heal. Arch Dermatol 135: 920-926.
31. Franks PJ, Moffatt CJ, Connolly M, Bosanquet N, Oldroyd MI, et al. (1995) Factors associated with Healing Leg Ulceration with High Compression. Age Ageing 24: 407-410.

32. Milic DJ, Zivic SS, Bogdanovic DC, Karanovic ND, Golubovic ZV (2009) Risk factors related to the failure of venous leg ulcers to heal with compression treatment. J Vasc Surg 49: 1242-1247.

33. Meaume S, Coullier D, Vin F (2005) Prognostic factors for venous ulcer healing in a non-selected population of ambulatory patients. J Wound Care 14: 31-34.

34. Simka M (2006) Clinical prognostic factors of healing of venous leg ulcers. Po Przegl Chir 78: 659-669.

35. Moffatt C, Doherty D, Smithdale R, Franks PJ (2010) Clinical predictors of leg ulcer healing. $\mathrm{Br} \mathrm{J}$ Dermatol 162: 51-58.

36. Davies CE, Hill KE, Newcombe RG, Stephens P, Wilson MJ, et al. (2007) A prospective study of the microbiology of chronic venous leg ulcers to reevaluate the clinical predictive value of tissue biopsies and swabs. Wound Repair Regen 15: $17-22$.

37. Lopez A, Phillips T (1998) Venous ulcers. Wounds 10: 149-157.

38. Rij AM van, Solomon C, Christie R (1994) Anatomic and physiologic characteristics of venous ulceration. J Vasc Surg 20: 759-764.

39. Padberg FT, Johnston MV, Sisto SA (2004). Structured exercise improves calf muscle pump function in chronic venous insufficiency: A randomized trial. $J$ Vasc Surg 39: 79-87.

40. Kan Y, Delis KT (2001) Hemodynamic effects of supervised calf muscle exercise in patients with venous leg ulceration: A prospective controlled study. Arch Surg 136: 1364-1369.

41. Dix F, Brooke R, McCollum C (2003). Venous disease is associated with an impaired range of ankle movement. Eur J Vasc Endovasc Surg 25: 556-561.

42. Schuren J, Bichel J (2013) Sub-bandage dynamics: Stiffness unravelled. Veins and Lymphatics 2: 2

43. Partsch H (2014) Compression for the management of venous leg ulcers: Which material do we have? Phlebology 29: 140-145.

44. Kimmel HM, Robin AL (2013) An evidence-based algorithm for treating venous leg ulcers utilizing the cochrane database of systematic reviews. Wounds 25 : 242-250.

45. Montero-Fernandez N, Serra-Rexach J (2013) Role of exercise on sarcopenia in the elderly. European Journal of Physical and Rehabilitation Medicine 49: 131-143.

46. Rasmussen JC, Aldrich MB, Tan IC, Darne C, Zhu B, et al.(2016) Lymphatic transport in patients with chronic venous insufficiency and venous leg ulcers following sequential pneumatic compression. Journal of Vascular Surgery: Venous and Lymphatic Disorders 4: 9-17.

47. Partsch H, Lee B (2014) Phlebology and lymphology-A family affair 645-647.

48. Mortimer PS (1995) Evaluation of lymphatic function: Abnormal lymph drainage in venous disease. Int Angiol 14: 32-35.

49. Hu D, Phan TT, Cherry GW, Ryan TJ (1998) Dermal oedema assessed by high frequency ultrasound in venous leg ulcers. Br J Dermatol 138: 815-820.

50. Nicolaides A (2000) Investigation of chronic venous insufficiency a consensus statement. Circulation 102: 126-163.

51. Alguire PC, Mathes BM (1997) Chronic venous insufficiency and venous ulceration. J Gen Intern Med 12: 374-383.

52. Mosti G (2014) Compression in leg ulcer treatment: Inelastic compression Phlebology 29: 146-152. 